\title{
Hardness Effects on Abrasive Flow Machining
}

\author{
Kursad Gov ${ }^{1}{ }^{*}$ - Omer Eyercioglu ${ }^{1}-$ Mehmed Veysel Cakir ${ }^{2}$ \\ ${ }^{1}$ Gaziantep University, Mechanical Engineering, Turkey \\ ${ }^{2}$ Vocational School of Kilis, Mechanical Department, Turkey
}

\begin{abstract}
Abrasive flow machining (AFM) is a manufacturing technique that uses the flow of a pressurized abrasive media to remove workpiece material. In comparison with other polishing technique, AFM is very efficient, suitable for the finishing of complex inner surfaces. In this paper, the effect of workpiece hardness on the AFM process has been investigated. An experimental study was carried out on AISI D2 tool steel hardened to 31, 45 and 55 HRC. The specimens were cut by using wire electro discharge machining (WEDM) and then finished with AFM. The results show that the white layer formed during WEDM is successfully removed by AFM in a few cycles. Although the surface quality is improved by AFM for all hardness groups, the results show that harder materials have more surface improvement than the softer ones.
\end{abstract}

Keywords: abrasive flow machining, surface roughness, wire electro discharge machining

\section{INTRODUCTION}

Abrasive flow machining (AFM) is a nontraditional machining process that was developed in the USA in the 1960s. AFM can be described as pressurized media passing through the surfaces to reach the desired surface quality. There are mainly two types of abrasive flow machines according to flow of media: one-way and two-way. The media consists of a type of polymeric carrier and abrasive particles that are $\mathrm{SiC}$, $\mathrm{Al} 2 \mathrm{O} 3$, diamond, etc.

AFM is used to deburr, polish, radius, and remove recast layers. Applications of AFM include the finishing of extrusion dies, medical implants and aerospace components. AFM can be successfully applied to different kinds of engineering materials from soft to hard, ferrous to nonferrous, and also metallic to nonmetallic. With the use of AFM, excellent surface finishes and close geometric tolerances can be attained economically. The media in the AFM process is used to polish difficult-to-reach areas, and to follow complex geometries and microholes.

The success of the AFM process depends on a number of process parameters that can be classified into three groups: AFM parameters, polishing media parameters and workpiece parameters. The AFM parameters are pressure, flow speed, number of cycles and machining time. The polishing media parameters are viscosity, temperature, abrasive material, mesh size and its concentration. The work-piece parameters are mainly the shape, the material hardness, the premachining process and surface texture orientation.

Rhodes [1] studied AFM processes and evaluated process parameters such as pressure, speed of the flow, volume of the media, types of abrasive, which affects the polishing of work pieces. Jain and Adsul
[2] studied the AFM process parameters, such as four kinds of mesh sizes and concentrations, the number of process cycles, different media flow speeds on aluminum and brass, simulating soft and hard materials, respectively. They concluded that dominant parameters are the number of process cycles, mesh size and abrasive concentration, and the effect of flow rate is less in comparison to the other AFM process parameters.

Jain et al. [3] studied the AFM media parameters (abrasive mesh size and concentration, temperature and viscosity) and found that the media viscosity increases with increasing abrasive concentration and decreases with the increase in mesh size and media temperature. Higher the viscosity results in high MRR and better surface roughness. Flether and Fioravanti [4] studied the rheology of the polyborosiloxane as media. They concluded that the concentration of abrasive is a more dominant parameter than grain size and polymer media.

Gorana et al. [5] studied the AFM media parameters such as media pressure, abrasive concentration and mesh size for silly putty (Dow Corning 3179) and silicon carbide. They were measured the acting forces on the specimens during process and showed the influence of media parameters on the resulting surface roughness. Agrawal et al. [6] used polyborosiloxane as media and predicted the viscoelastic properties of media such as viscosity, creep compliance and bulk modulus. Loveless et al. [7] compared AFM process on the various machined surfaces obtained from grinding, milling, turning, and wire electro-discharge machine (WEDM) operations. They reported that the best improvement in the surface quality was obtained on the WEDMed surfaces.

Kenda et al. [8] studied the influence of process parameters on surface integrity. They used EDM AISI 
D2 pre-machined hardened tool steel as a work piece and reported that AFM is capable of removing an EDM damaged surface and significantly improving the surface roughness as well as of inducing high compressive residual stresses onto the machined surface. Bähre et al. [9] developed a setup to measure the axial forces under applied media pressure during the AFM of AISI 4140 steel. They presented the influence of media pressure on the surface quality and form tolerance.

However, there is only one study available in the literature [2] considering the effect of the hardness of the workpiece material, but the study [2] was carried out for brass and aluminum as hard and soft materials, respectively. Therefore, it is necessary to show the influence of the hardness of the steel on the AFM process. This paper is focused on the effect of workpiece hardness on the AFM process. Three groups of AISI D2 tool steel (hardened to 31, 45 and 55 HRC) were cut by WEDM. The surface roughness values were measured and scanning electron microscope (SEM) images were taken from the surfaces before and after AFM for various processing cycles. The improvement of the surfaces with respect to the hardness was observed.

\section{EXPERIMENTAL WORKS}

\subsection{Workpiece Material}

The workpiece is made of the heat treated AISI D2 tool steel. $10 \times 40 \times 500 \mathrm{~mm}$ slabs were cut from the same stock and heat treated to 31,45 and 55 HRC. The specimens were cut from the slabs by using WEDM to $10 \times 40 \times 10 \mathrm{~mm}$. The WEDM parameters were kept constant for all specimens to ensure the presurface characteristics of the specimens. The surface roughness values of the specimens after WEDM are given in Table $1\left(R_{a}, R_{q}, R_{z}\right)$.

\subsection{The Abrasive Flow Machine}

The AFM machine used in the study is given in Fig. 1; it was designed and constructed in the Advanced Machining Center of The University of Gaziantep. It is a one-way AFM and the specifications of the machine are given in Table 2. The machine has three units: the main frame, the control system, and the hydraulic unit. The hydraulic unit ensures adequate movement and media pressure that can be manually configured. The control system is designed to control the volume of polishing media and the number of cycles. A cycle in one-way AFM is composed of the filling stage of the cylinder and the complete stroke of the piston. Thus, the cycle time depends on the flow rate (i.e. piston speed) and one cycle in the experimental study takes one minute.

Table 1. Surface roughness values of the specimens before AFM

\begin{tabular}{ccccc}
\hline Hardness & Specimen No & Ra $[\mu \mathrm{m}]$ & $\mathrm{Rz}[\mu \mathrm{m}]$ & $\mathrm{Rq}[\mu \mathrm{m}]$ \\
\hline \multirow{3}{*}{$31 \mathrm{HRC}$} & $\mathrm{A} 13$ & 2.5 & 10.34 & 2.96 \\
\cline { 2 - 5 } & $\mathrm{A} 14$ & 2.4 & 12.88 & 2.52 \\
\cline { 2 - 5 } & $\mathrm{A} 15$ & 2.4 & 11.42 & 2.48 \\
\cline { 2 - 5 } & $\mathrm{A} 16$ & 2.5 & 10.09 & 2.61 \\
\cline { 2 - 5 } & $\mathrm{A} 17$ & 2.5 & 9.17 & 2.84 \\
\cline { 2 - 5 } & $\mathrm{A} 18$ & 2.5 & 12.33 & 3.19 \\
\hline \multirow{5}{*}{$45 \mathrm{HRC}$} & $\mathrm{B} 13$ & 2.4 & 14.2 & 2.85 \\
\cline { 2 - 5 } & $\mathrm{B} 14$ & 2.6 & 11.93 & 2.42 \\
\cline { 2 - 5 } & $\mathrm{B} 15$ & 2.4 & 11.5 & 2.38 \\
\cline { 2 - 5 } & $\mathrm{B} 16$ & 2.6 & 9.34 & 2.93 \\
\cline { 2 - 5 } & $\mathrm{B} 17$ & 2.4 & 8.49 & 2.77 \\
\hline \multirow{3}{*}{ 55 HRC } & $\mathrm{B} 18$ & 2.4 & 10.42 & 2.11 \\
\cline { 2 - 5 } & $\mathrm{C} 13$ & 2.4 & 12.05 & 3.02 \\
\cline { 2 - 5 } & $\mathrm{C} 14$ & 2.6 & 11.65 & 2.57 \\
\cline { 2 - 5 } & $\mathrm{C} 15$ & 2.4 & 12.19 & 2.52 \\
\cline { 2 - 5 } & $\mathrm{C} 16$ & 2.4 & 9.9 & 2.75 \\
\cline { 2 - 5 } & $\mathrm{C} 17$ & 2.4 & 10 & 2.88 \\
\cline { 2 - 5 } & $\mathrm{C} 18$ & 2.5 & 12.11 & 3.14 \\
\hline
\end{tabular}

Table 2. Abrasive flow machine

\begin{tabular}{ll}
\hline Machine Specification & \\
\hline Pump Pressure & 350 to $1000 \mathrm{psi}$ (24 to 200 bar) \\
\hline Media capacity & 2 litre \\
\hline Stroke & $250 \mathrm{~mm}$ \\
\hline Bore diameter & $120 \mathrm{~mm}$ \\
\hline
\end{tabular}

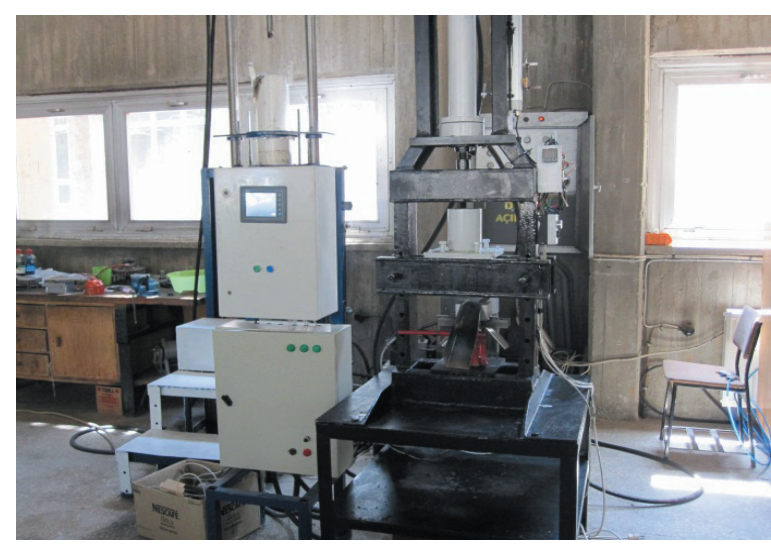

Fig. 1. Abrasive flow machine

\subsection{Polishing Media}

The polishing media used in the experiments is a mixture of a polymer-based carrier and abrasive 
particles. The specifications of the medium are summarized in Table 3.

Table 3. Polishing Media

\begin{tabular}{ll}
\hline Parameters & \\
\hline Viscosity & 60 Pa.s \\
\hline Abrasive type & Al203 \\
\hline Mesh size & 180 \\
\hline Abrasive concentration & $70 \%$ wt. \\
\hline
\end{tabular}

\subsection{Experimental Procedure}

The experiments were performed on the three groups of specimens (31, 45 and 55 HRC). A fixture (see Fig. 2) was used to hold the specimens allowing the flow of polishing media through the WEDMed surfaces with an opening of $2 \times 20 \mathrm{~mm}$. A total of $2000 \mathrm{~g}$ of polishing media was flowing through in one cycle and the experiments were carried out for 1, 3, 5, 10, 20 and 100 cycles. The machining parameters were chosen according to the successful results of preliminary studies. These parameters are generally depending on the capacity of the machine, viscosity and abrasive concentration of the media, geometry of the surface and the opening. The AFM pressure was $10 \mathrm{MPa}$ and the flow rate was $50 \mathrm{~g} / \mathrm{s}$. The experiments were repeated for three specimens in each condition and the averages of the 18 surface roughness measurements were taken. A Mitutoyo SJ 400 surface measuring machine was used and the cut of length was chosen as $0.8 \mathrm{~mm}$. The SEM images of the surfaces were taken by using a Jeol JSM-6390 LV electron microscope.

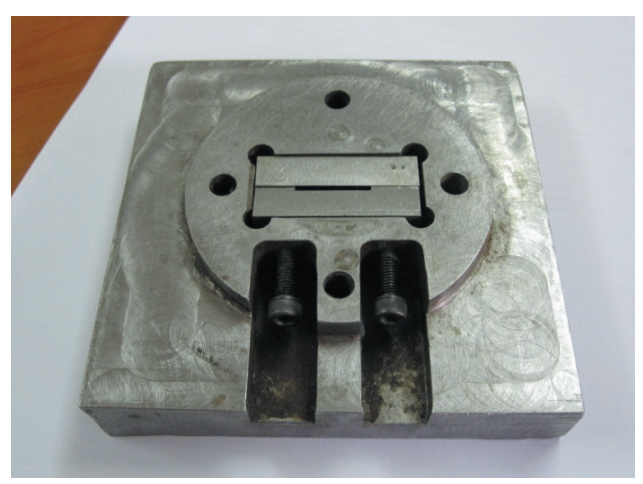

Fig. 2. Fixture with workpiece

\section{RESULTS AND DISCUSSION}

\subsection{Measurements of Surface Roughness}

The results of the selected roughness parameters $\left(R_{a}, R_{q}, R_{z}\right)$ for three hardness groups are presented in Fig. 3; the values are the average of 18 measures along and perpendicular to the flow directions. As seen from the figures, the surface roughness decreased with increase in number of cycles for all hardness values. The results show that the surface roughness of the WEDMed surfaces significantly changed in first 20 cycles and then settled to a saturated level gradually. The surface roughness after 50 cycles is decreased slightly. Although the trends are similar for all hardness groups, the final surface roughness values are different. The harder the workpiece is better the surface roughness according to the final measures. This result has to be explained together with the SEM images of the surfaces which are given in the following section. It should be observed that these surface roughness values were obtained by using 180 mesh abrasives, better finishing may be obtained low abrasive sizes with subsequent operations.
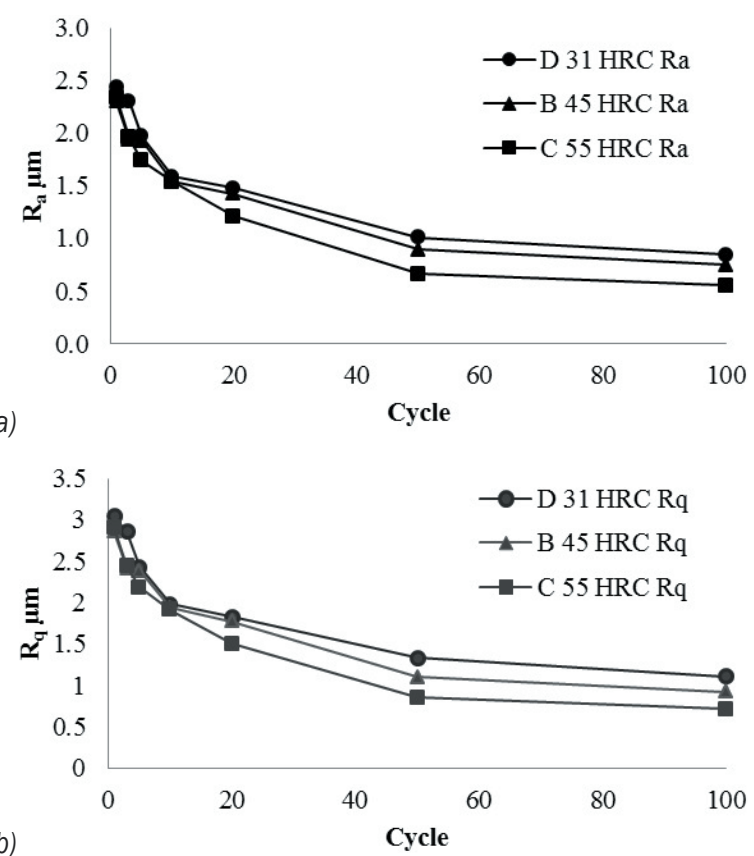

b)

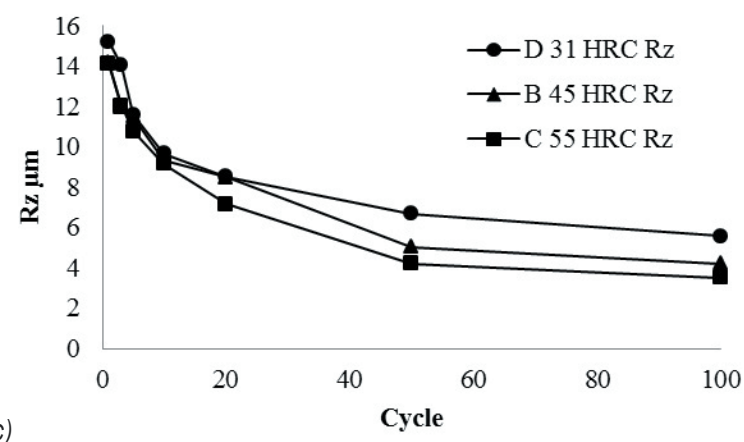

Fig 3. Change in surface roughness with respect to AFM cycles; a) $R_{a}$, b) $R_{q}$ and c) $R_{z}$ 

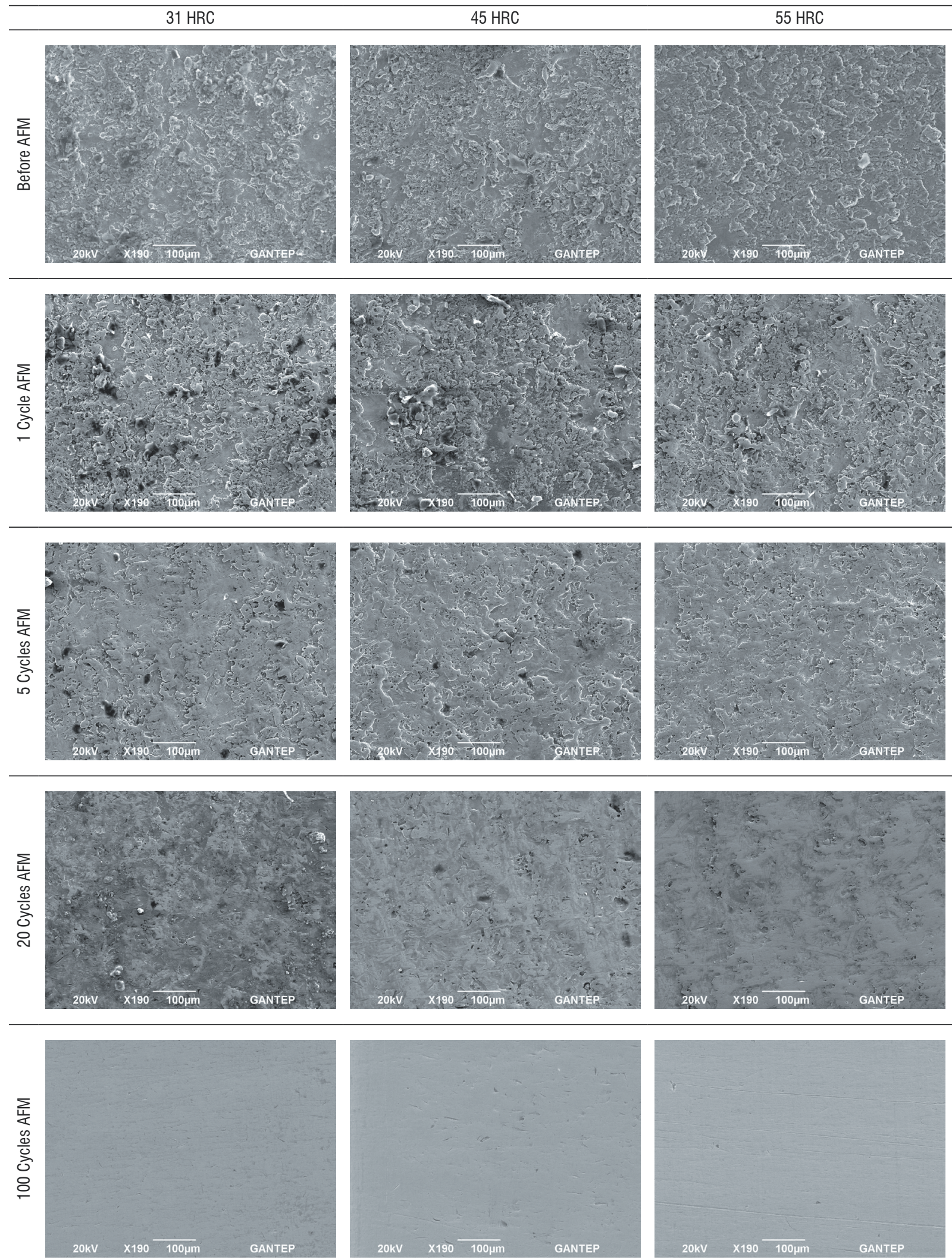

Fig. 4. SEM images of AFM at $190 \times$ magnification 


\subsection{SEM Images}

SEM images of taken from the surfaces of the specimens are given in Fig. 4. During the wire EDM process, rapid heating and cooling occurs, and causes a heat-affected layer on the machined surface to form. This layer is generally formed by the sticking of the non-removed debris and also the depth of material affected from high temperature. The top of the layer consists of a re-solidified layer and has a re-cast structure. The recast layer seems to have a white colour when examined with a microscope and thus it is commonly referred to as "the white layer". The white layer is very hard and brittle, and has microcracks.

In Fig. 5, Microscopic photographs of the white layers for three groups of specimens are given. Due to the nature of the EDM, the white layer thickness is taken as an average value. The average white layer thickness values of the three groups of specimens are near to each other (about $22 \mu \mathrm{m}$ ). In the first five cycles of the AFM process, the white layer is removed for all specimens. This can be seen from Fig. 5, as the improvement in the surface roughness values of all groups are similar up to five AFM cycles.

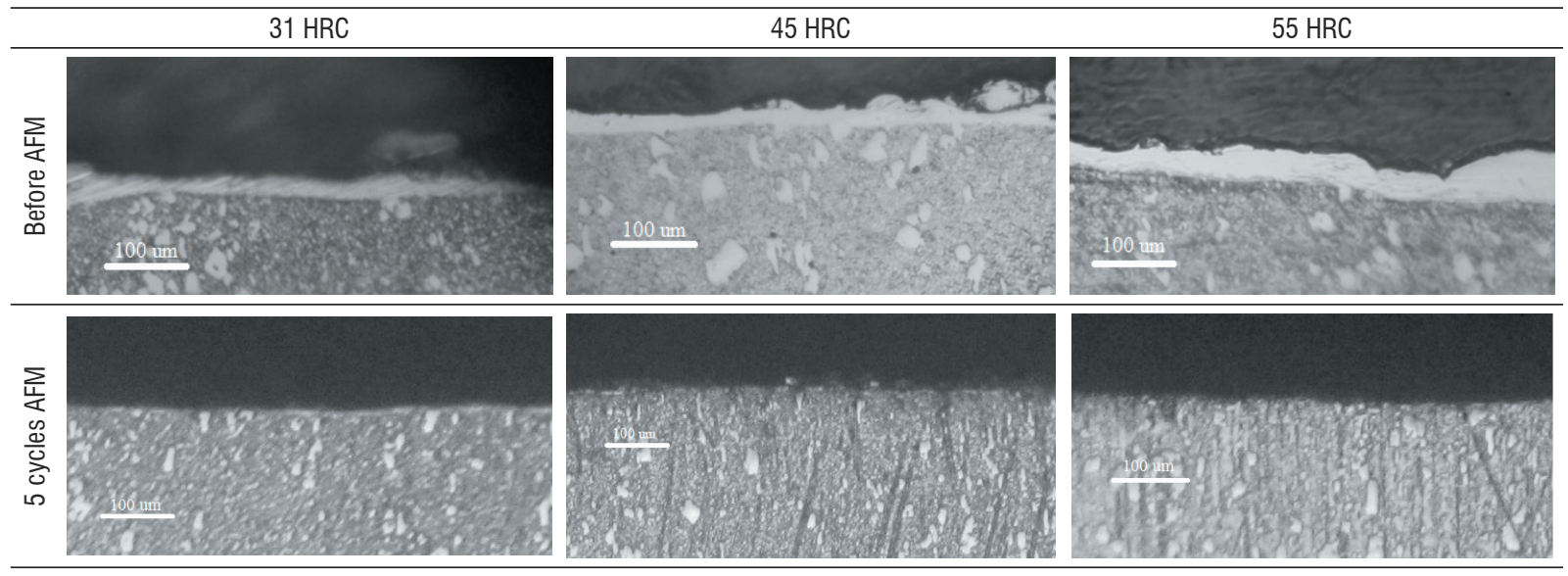

Fig 5. Microscopic photographs of before and after AFM process
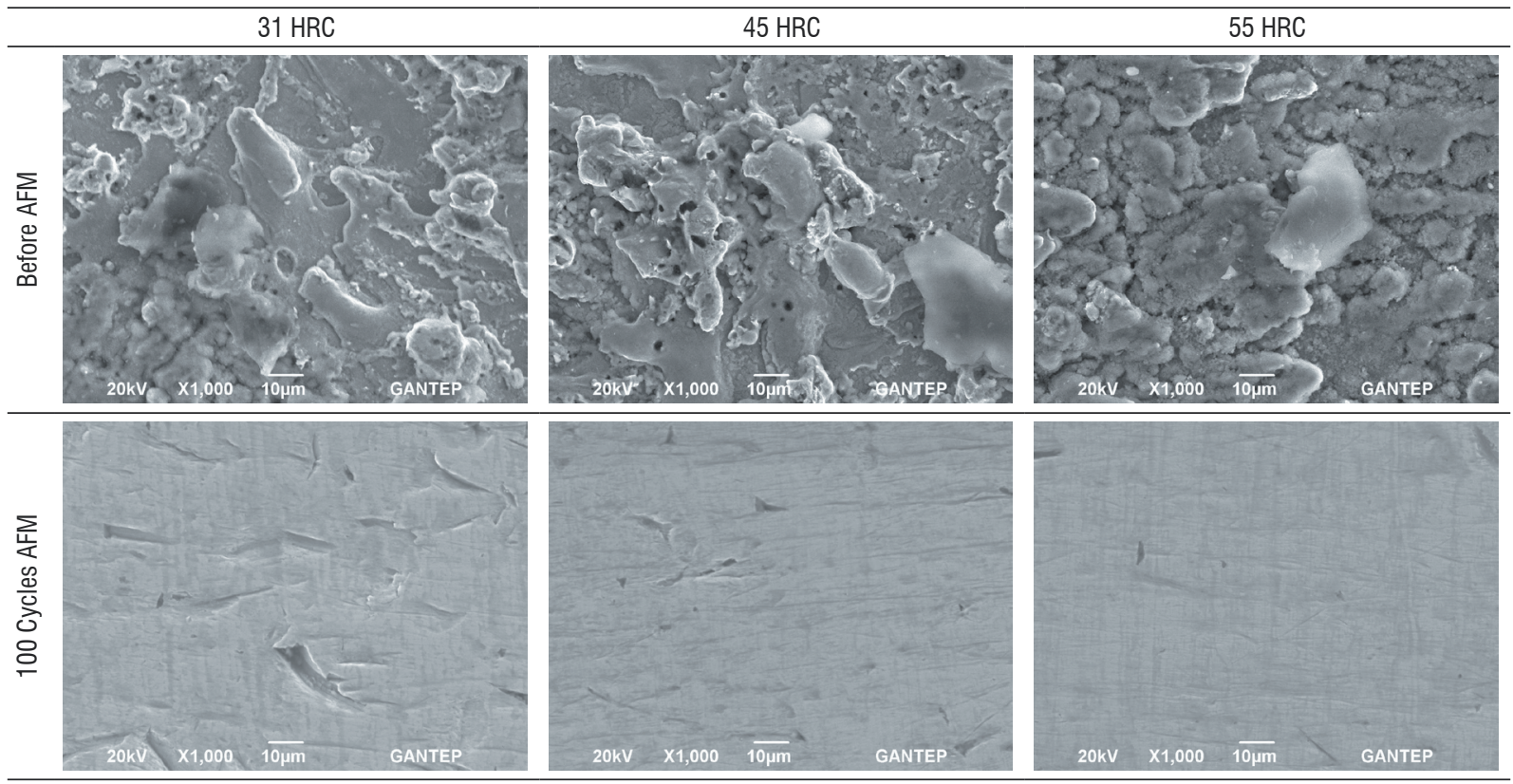

Fig 6. SEM images of AFM at $1000 \times$ magnification 
After removal of the white layer, abrasion the behaviors of the three hardness groups were changed. In the softer specimens ( $31 \mathrm{HRC})$, the indentation of the abrasive particles to the surfaces was observed. For the harder group (55 HRC), smearing and plowing were less frequent and the final surface roughness is better. The differences between the initial and final surfaces of the three groups of specimens can be seen from Fig. 6 at a magnification ratio of 1000.

\section{CONCLUSIONS}

In this paper, the effect of hardness of WEDMed AISI D2 tool steel on abrasive flow machining was investigated. From the experimental results, the following conclusions have been derived:

- The white layer formed during WEDM is successfully removed by AFM in a few cycles. The removal of this layer eliminates surface cracks and thus the fatigue strength may increase.

- The surface quality is improved by AFM for all hardness groups. The results show that the surface roughness of the WEDMed surfaces significantly changed in the first 20 cycles and then settled to a saturated level gradually. The surface roughness after 50 cycles is decreased slightly.

- Although the trends of surface roughness measurements are similar for all hardness groups, the results show that harder material has more surface improvement than the softer ones.

\section{ACKNOWLEDGEMENTS}

The authors would like to thank Gaziantep University Scientific Research Council (BAP) due to financial support to this research.

\section{REFERENCES}

[1] Rhodes, L. (1991). Abrasive flow machining: A case study. Journal of Materials Processing Technology, vol. 28, no. 1-2, p 107-116, DOI:10.1016/09240136(91)90210-6.

[2] Jain, V.,K., Adsul, S.,G. (2000). Experimental investigation into abrasive flow machining. International Journal of Machine Tools and Manufacture, vol. 40, no. 7, p. 1003-1021, DOI:10.1016/S0890-6955(99)00114-5.

[3] Jain, V.K., Ranganatha, C., Muralidhar, K. (2001). Evaluation of Rheological properties of medium for AFM process. Machining Science and Technology: An International Journal, vol. 5, no. 2, p, 151-170, DOI:10.1081/MST-100107841.

[4] Flether, A.J., Fioravanti, A. (1996). Polishing and honing processes: An investigation of the thermal properties of mixture of polyborosiloxane and silicon carbide abrasive. Proceedings of the Institution of Mechanical Engineers. Part C: Journal of Mechanical Engineering Science, vol. 210, no. 3, p. 255-265, DOI:10.1243/PIME_PROC_1996_210_195_02.

[5] Gorana, V.,K., Jain, V.,K., Lal, G.,K, (2004). Experimental investigation into cutting forces and active grain density during abrasive flow machining. International Journal of Machine Tools and Manufacture, vol. 44, no 2-3, p. 201-211, DOI:10.1016/j.ijmachtools.2003.10.004.

[6] Agrawal, A., Jain, V.K., Muralidhar, K. (2005). Experimental determination of viscosity of abrasive flow machining media. International Journal of Manufacturing Technology and Management, vol. 7, no. 2-4, p. 142-156, DOI:10.1504/IJMTM.2005.006828.

[7] Loveless, T.R., Williams, R.E., Rajurker, K.P. (1994). A study of the effects of abrasive-flow finishing on various machined surfaces. Journal of Materials Processing Technology, vol. 47, no. 1-2, p. 133-151, DOI:10.1016/0924-0136(94) 90091-4.

[8] Kenda, J., Pusavec, F., Kermouche, G., Kopac, J. (2011). Surface integrity in abrasive flow machining of hardened tool steel AISI D2. Procedia Engineering, vol. 19, p. 172-177, DOI:10.1016/j.proeng.2011.11.097.

[9] Bähre, D., Brünnet, H., Swat, M. (2012). Investigation of one-way abrasive flow machining and in-process measurement of axial forces. Fifth CIRP Conference on High Performance Cutting, vol. 1, p. 419-424, DOI:10.1016/j.procir.2012.04.075. 